\title{
Discapacidad en el trastorno obsesivo compulsivo. Más allá de un diagnóstico clínico
}

\author{
Giovani Díaz-Gervasi ${ }^{1}$ \\ Claudia Cahui-Ramírez
}

RESUMEN: Reporte de caso clínico de una paciente adulta con un trastorno obsesivo compulsivo y un nivel moderado a severo de discapacidad derivado del mismo. Se abordó el caso desde una perspectiva biopsicosocial bajo un enfoque psicoterapéutico cognitivo conductual. Al realizar la evaluación y diagnóstico se hallaron pensamientos obsesivos, compulsiones y síntomas depresivos intensos. Posteriormente se aplicó un plan de intervención psicológica en dos fases orientado a disminuir la intensidad de los síntomas clínicos. Se incentivó la funcionalidad en las áreas individual, familiar, social, de realización personal y productividad. El programa se ejecutó durante dos años y medio con sesiones continuas y de seguimiento para verificar y reforzar los cambios. Finalizado el proceso se obtuvo una mejora sustancial en el afronte de los pensamientos obsesivos y la reducción de conductas compulsivas. Se logró disminuir sustancialmente el nivel de discapacidad mejorando la relación con su entorno familiar y social. También se logró su inserción laboral y buen desempeño. El abordaje integral y no solo centrado en el diagnóstico clínico de la persona permite una intervención psicológica eficaz y acorde a las necesidades específicas del caso. Las técnicas cognitivo-conductuales demuestran ser útiles en el tratamiento del TOC, comorbilidades, en el incremento de la calidad de vida y productividad personal.

PALABRAS CLAVE: Discapacidad mental, Trastorno obsesivo compulsivo; Terapia cognitivo-conductual; Perspectiva biopsicosocial.

Citar como: Díaz G, Cahui C. Discapacidad en el trastorno obsesivo compulsivo. Más allá de un diagnóstico clínico. CASUS. 2017;2(3):200-208.

${ }^{1}$ Universidad Católica Sedes Sapientiae. Magister en Psicología clínica. ¿Universidad Católica Sedes Sapientiae. Licenciada en Psicología. 


\section{INTRODUCCIÓN}

Los trastornos mentales pueden afectar la salud mental, física y social de la persona. En muchos casos los pacientes presentan de forma progresiva deterioro cognitivo y niveles significativos de discapacidad funcional (1). De esta manera, los diversos desórdenes mentales pueden afectar también la calidad y el tiempo de vida. Cifras internacionales revelan que los trastornos de ansiedad (entre los que está incluido el Trastorno Obsesivo Compulsivo, TOC) afectan a 260 millones de personas que pueden experimentar una disminución en su capacidad laboral, organizativa y productiva (2). Es así que, las personas bajo esta condición tienen entre 6 y 7 veces más probabilidades de estar desempleadas y reducir su tiempo de vida hasta en 20 años (3).

Un trastorno obsesivo compulsivo va más allá de las preocupaciones, creencias supersticiosas y actos ritualísticos que pueden ser comunes en la vida de algunas personas. En el TOC las ideas intrusivas aparecen reiteradamente en el contenido normal del pensamiento. Estos pensamientos son incontrolables, interfiriendo significativamente con las actividades cotidianas de la persona en cualquier ámbito (2). Asimismo, existe inquietud, aprensión, preocupación, y conductas repetitivas denominadas compulsiones, dirigidas a reducir la alta ansiedad asociada (4). Por otra parte, la persona intenta sin éxito ignorar y suprimir estos pensamientos o impulsos que acarrean un significativo malestar psicológico (4).

Con frecuencia este trastorno puede promover en el paciente el desarrollo de sintomatología y trastornos depresivos e ideación o actos suicidas (5). Estos síntomas pueden generar condiciones de discapacidad en la persona que lo padece. La discapacidad originada por un trastorno mental acarrea la pérdida progresiva de la funcionalidad de la persona a nivel individual, social, familiar, laboral o educativo $(6,7)$. En el 2012 un estudio realizado en Lima y Callao halló un $10.3 \%$ de discapacidad debida a problemas mentales en población adulta, $17.9 \%$ en adolescentes y $18.9 \%$ en los adultos mayores. A su vez se señaló que un $0.2 \%$ (casi medio millón de personas) presentaría el TOC alguna vez en su vida (6). Sumado a ello se ubica a nivel nacional como el trastorno mental que genera mayor discapacidad a nivel moderado según estándares internacionales (8).

En el Perú hay poca información adecuadamente documentada sobre la intervención psicológica en el
TOC y el efecto que ejerce sobre la funcionalidad del sujeto. Considerando las consecuencias que acarrea la discapacidad mental es necesario reportar los programas de intervención psicológica orientados a disminuir los síntomas y el incremento de la adaptación y productividad de los afectados.

Añadido a lo anterior, tradicionalmente se enfatiza el diagnóstico nosológico como el factor más importante para la evaluación e intervención en la enfermedad mental. Sin embargo, se hace evidente la necesidad de tomar en cuenta los factores psicológicos, sociales y experienciales que también sirven al profesional de la salud para brindar una atención eficaz. De esta manera no se reduce a la persona a su diagnóstico clínico desde una perspectiva psicopatológica. En este sentido, el presente reporte de caso tiene como propósito documentar el abordaje integral durante el proceso de evaluación, diagnóstico e intervención psicológica desde la perspectiva cognitivo-conductual en una mujer adulta con la presencia de TOC, trastorno depresivo y con un nivel de discapacidad de moderada a severa.

\section{PRESENTACIÓN DEL CASO}

Paciente adulta que asistió sola a consulta, de 28 años, soltera y de religión Católica. Presentó contextura delgada, aliño e higiene personal adecuados. Se encontró lúcida, orientada en espacio, tiempo y persona. Manifestó pensamiento de curso lógico y de contenido obsesivo, depresivo y ansioso. Su estado anímico denotó ansiedad y tristeza. Asimismo, refirió que no trabajaba ni estudiaba porque no se "sentía capaz" por los pensamientos constantes que no permitían su concentración en actividades laborales y acusó una intensa abulia y anhedonia llegando a la ideación suicida.

Refirió que venía al Servicio de Psicología por decisión propia y que seguía tratamiento en un hospital de salud mental en el cual le diagnosticaron solamente depresión, por lo cual se encontraba recibiendo tratamiento antidepresivo desde hace tres años. Sin embargo, revelaba no sentir alivio. Señaló que no tenía interés de colaborar en las actividades del hogar, en su aliño personal, en buscar un empleo, y tener momentos agradables con amigos o personas conocidas. Pasaba la mayor parte del tiempo en su habitación "pensando" sobre el "no olvidar lo que estaba pensando antes o de lo que tenía que hacer luego" y consecuentemente a estos pensamientos "escribo todo en un cuaderno para no olvidarme de nada". Así mismo, refirió que tenía 
"pensamientos malos sobre Dios" a manera de "insultos o frases impropias" que le hacían sentir culpable y "sin ganas de hacer nada". Estos pensamientos se repetían "la mayor parte del día" a pesar de que ella trataba de controlarlos o cuestionarlos y reconocía que los pensamientos eran absurdos, pero le generaban "mucho nerviosismo". "Paso casi todo el tiempo pensando y apuntándolo todo en un cuaderno". Señaló además que tenía problemas para conciliar el sueño y que olvidaba algunas cosas que sucedieron recientemente o que le encargaban hacer en casa. "Ya no se qué hacer para dejar de pensar en esto, siento que las pastillas que tomo no me hacen bien".

\section{EVALUACIÓN Y DIAGNÓSTICO}

El proceso de evaluación y diagnóstico se realizó en base a una entrevista inicial, la historia clínica y la aplicación de pruebas psicológicas que abarcaron las áreas de organicidad, TOC, inteligencia, personalidad, pensamiento, afectividad y discapacidad (ver tabla 1).

Tabla 1. Áreas evaluadas y pruebas aplicadas

\begin{tabular}{|c|c|}
\hline Áreas & Pruebas psicológicas \\
\hline Organicidad & $\begin{array}{l}\text { - Test Guestáltico Visomotor } \\
\text { de Bender } \\
\text { - Test de Retención Visual de } \\
\text { Benton } \\
\text { - Escala de memoria de } \\
\text { Wechsler forma I }\end{array}$ \\
\hline Inteligencia & $\begin{array}{l}\text { - Escala de inteligencia para } \\
\text { adultos de Wechsler }\end{array}$ \\
\hline Pensamiento & $\begin{array}{lll}\text { - } & \text { Test de asociación de } \\
& \text { palabras de Yung }\end{array}$ \\
\hline Personalidad & $\begin{array}{l}\text { - Inventario multifásico de la } \\
\text { - } \quad \text { Inversonalidad MMPI forma R } \\
\text { de Eysenck y Eysenck }\end{array}$ \\
\hline Afectividad & $\begin{array}{l}\text { - Inventario de depresión de } \\
\text { Beck } \\
\text { - } \quad \text { Escala de autovaloración de } \\
\text { la ansiedad de Zung }\end{array}$ \\
\hline TOC & $\begin{array}{llr}\text { - Escala Yale } & \text { Brown para } \\
\text { trastorno } & \text { obsesivo } \\
\text { compulsivo } & \end{array}$ \\
\hline Discapacidad & $\begin{array}{ll}\text { - Escala abreviada de } \\
\text { discapacidad e inhabilidad }\end{array}$ \\
\hline
\end{tabular}

Por otro lado, se evaluaron los niveles de discapacidad partiendo de la Escala abreviada de discapacidad e inhabilidad categorizada en cuatro niveles: ninguna, leve, moderada, severa. La misma abarca áreas de discapacidad e inhabilidad, tales como: cuidado personal, funcionamiento ocupacional, familiar, social y organizativo. Estas áreas comprenden los siguientes aspectos:

- Cuidado personal: se evaluaron las limitaciones para su desempeño en el aseo y aliño personal, la limpieza y dedicación en su vestimenta.

- Funcionamiento ocupacional: se consideraron aquellas barreras para su desenvolvimiento en el área laboral y las tareas del hogar.

- Funcionamiento familiar: se orientó a evaluar su desempeño en el rol familiar y la comunicación.

- Funcionamiento social: se analizaron las limitaciones para su interacción en las relaciones interpersonales y habilidades sociales con familiares, amigos, vecinos, etc.

- Funcionamiento organizativo: se incluyeron aquellas barreras para su desempeño en la organización y ejecución de las actividades cotidianas en general.

Diagnóstico psicológico: Se halló de base un trastorno obsesivo-compulsivo, un trastorno depresivo de moderado a severo y un nivel de discapacidad de moderada a severa.

\section{INTERVENCIÓN PSICOLÓGICA}

El caso fue abordado desde una perspectiva biopsicosocial y bajo un enfoque cognitivo-conductual y sus técnicas de intervención. Esta perspectiva aborda a la persona considerando no solo el problema psicológico u orgánico, sino también los aspectos sociales, experienciales y culturales (9). El enfoque cognitivo conductual tiene sus bases en el procesamiento de la información y la teoría del aprendizaje. El aspecto cognitivo se fundamenta en la teoría de Beck (10) y sus tres supuestos: la triada cognitiva (pensamientos negativos respecto a sí mismo, las experiencias y al futuro), los esquemas mentales (cogniciones arraigadas en el sujeto) y los errores cognitivos (ideas irreales que generan sentimientos de malestar y conductas desadaptativas).

Entre las técnicas de intervención cognitivo conductuales se aplicaron: la relajación muscular 
progresiva de Jacobson y la técnica de imágenes inducidas para el control de la ansiedad. La parada de pensamiento, la reatribución de la culpa, la aserción encubierta, el registro cognitivo-conductual, la intención paradójica y la desdramatización del pensamiento para el control y disminución de los pensamientos obsesivos y síntomas depresivos (10).

El proceso psicoterapéutico se llevó a cabo durante un período de dos años y medio. El mismo se dividió en dos etapas según la frecuencia de las sesiones:

a. Etapa I de tratamiento continuo: las sesiones se desarrollaron dos veces por semana, posteriormente una semanal y luego quincenalmente.

b. Etapa II de seguimiento: la frecuencia varió desde dos veces al mes al inicio, hasta una vez cada seis meses de acuerdo a lo requerido. Algunas sesiones de seguimiento breves se realizaron vía telefónica hasta un año después del tratamiento.

Las estrategias de intervención psicológica estuvieron agrupadas según objetivos preestablecidos relacionados al afronte y reducción de los pensamientos obsesivos, compulsiones y sintomatología ansioso-depresiva (ver tabla 2).

\section{Tabla 2. Programa de intervención}

\begin{tabular}{|c|c|}
\hline $\begin{array}{l}\text { Estrategias de } \\
\text { intervención }\end{array}$ & Objetivo \\
\hline $\begin{array}{ll}\text { - } & \text { Interrupción de } \\
& \text { pensamiento } \\
\text { - } & \text { Desdramatización o } \\
& \text { flecha descendente } \\
\text { - } & \text { Intención paradójica }\end{array}$ & $\begin{array}{l}\text { Afronte y } \\
\text { disminución de } \\
\text { pensamientos } \\
\text { obsesivos. }\end{array}$ \\
\hline $\begin{array}{ll}\text { - } & \text { Prevención de } \\
& \text { respuestas } \\
\text { - Uso de conductas } \\
\text { alternativas }\end{array}$ & $\begin{array}{l}\text { Disminución de } \\
\text { compulsiones. }\end{array}$ \\
\hline $\begin{array}{l}\text { Terapia cognitiva } \\
\text { (modelo de Aaron } \\
\text { Beck) }\end{array}$ & $\begin{array}{l}\text { Modificación de } \\
\text { pensamientos } \\
\text { negativos e } \\
\text { irreales. }\end{array}$ \\
\hline $\begin{array}{ll}\text { - } & \text { Entrenamiento en } \\
& \text { habilidades sociales }\end{array}$ & $\begin{array}{l}\text { Desarrollar } \\
\text { conductas que } \\
\text { incrementen la } \\
\text { eficacia social. }\end{array}$ \\
\hline $\begin{array}{l}\text { - Entrenamiento en } \\
\text { técnicas de relajación }\end{array}$ & $\begin{array}{l}\text { Reducir niveles de } \\
\text { ansiedad. }\end{array}$ \\
\hline
\end{tabular}

\section{TRATAMIENTO}

El total de tiempo de la intervención fue de 21 meses sin contar con las dos últimas sesiones de seguimiento semestral. La programación de sesiones por área se llevó a cabo en un total de 76 sesiones, la duración de cada una fue de $45 \mathrm{~min}$. Las mismas fueron agrupadas de la siguiente manera:

- 32 sesiones destinadas a mejorar el bienestar individual.

- 13 sesiones dirigidas a desarrollar habilidades de comunicación a nivel familiar.

- 15 sesiones para desarrollar habilidades sociales.

- 9 sesiones de apoyo relacionadas con la productividad y realización personal.

- 7 sesiones de seguimiento para verificar la consistencia y permanencia de los cambios.

Las sesiones fueron estructuradas en cinco fases: el inicio (bienvenida y retroalimentación); la explicación del tema a abordarse y las técnicas a aplicarse; la puesta en práctica de la técnica; la elaboración conjunta de la conclusión de la sesión y por último, el acuerdo psicoterapéutico para las actividades prácticas en situaciones concretas. Asimismo, el proceso de intervención abordó las siguientes áreas: el bienestar individual, la familia, el entorno social, la productividad y la realización personal (ver tabla 3).

\section{RESULTADOS}

A través de la intervención psicológica se lograron resultados relacionados a cada área de intervención:

- A nivel individual se logró la deshabituación, el control de los pensamientos obsesivos y la disminución de la carga emocional que estos producían. Otro aspecto fue la reducción significativa de la sintomatología depresiva. Asimismo, se fortaleció la capacidad de tolerancia y afronte a las críticas en diferentes contextos, incrementando su resiliencia ante a situaciones que le resultaban limitantes. Por otra parte, la paciente solicitó voluntariamente la disminución de la administración de psicofármacos, culminando el proceso psicoterapéutico sin la necesidad de tomarlos. 
Tabla 3. Áreas de intervención

\begin{tabular}{|c|c|}
\hline Áreas & Objetivos \\
\hline $\begin{array}{l}\text { Bienestar } \\
\text { individual }\end{array}$ & $\begin{array}{l}\text { - } \text { Reducir los síntomas } \\
\text { obsesivo-compulsivos y } \\
\text { ansioso-depresivos. } \\
\text { Reestructurar las } \\
\text { cogniciones erróneas } \\
\text { sobre sí misma, sus } \\
\text { experiencias y su } \\
\text { entorno. }\end{array}$ \\
\hline $\begin{array}{l}\text { Bienestar } \\
\text { familiar }\end{array}$ & $\begin{array}{l}\text { - } \text { Minimizar los } \\
\text { pensamientos negativos y } \\
\text { actitudes no productivas } \\
\text { en el hogar. } \\
\text { Mejorar la comunicación y } \\
\text { relación hacia la madre y } \\
\text { hermana. }\end{array}$ \\
\hline $\begin{array}{l}\text { Entorno } \\
\text { social }\end{array}$ & 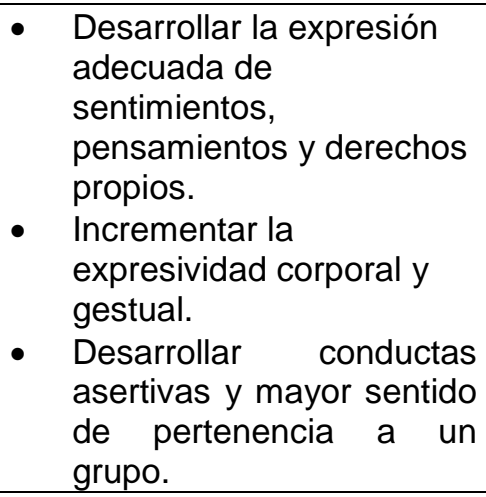 \\
\hline $\begin{array}{l}\text { Productividad } \\
\text { y realización } \\
\text { personal }\end{array}$ & $\begin{array}{l}\text { - Incrementar la disposición } \\
\text { hacia la realización de } \\
\text { actividades. } \\
\text { - Incentivar la realización } \\
\text { personal y sentido de } \\
\text { utilidad a través del } \\
\text { trabajo. } \\
\text { Establecer metas } \\
\text { realistas en cuanto a su } \\
\text { situación personal, } \\
\text { económica y social. }\end{array}$ \\
\hline
\end{tabular}

- En la esfera familiar se logró un cambio positivo de actitud en la madre y hermana hacia la paciente. Las relaciones intrafamiliares mejoraron considerablemente en cuanto a la calidad de la comunicación, confianza y unidad familiar. Esto promovió en la paciente pensamientos y sentimientos de seguridad, pertenencia y protección.

- En el aspecto social la paciente desarrolló un estilo asertivo de comunicación y expresión de emociones y sentimientos. De esta manera, participó en algunas jornadas comunitarias de su parroquia y compromisos sociales.

- En la productividad y realización personal se alcanzó un mejor desempeño psicosocial y laboral. Fue a través del trabajo que la paciente confrontó sus creencias negativas con la realidad y reestructuró su pensamiento. Esto motivó una visión más útil de sí misma y del tiempo, logrando desenvolverse eficientemente en el trabajo. Así mismo, en el aspecto organizativo, se incrementó el nivel de funcionalidad y el desempeño en la ejecución de tareas cotidianas que antes le resultaban difíciles de concretar.

\section{DISCUSIÓN}

El presente reporte de caso clínico tuvo como objetivo documentar la reducción de la sintomatología obsesiva y depresiva que generaban niveles de discapacidad de moderado a severo en la paciente, a través de la terapia cognitivo conductual (TCC). Se logró una mayor adaptación y funcionalidad en la misma. A nivel individual se consiguió la deshabituación y control de los pensamientos obsesivos y conductas compulsivas. En el ámbito familiar se mejoró la relación, la comunicación y el sentido de pertenencia. En el aspecto social se logró un mejor desempeño a través del desarrollo de habilidades sociales. En la productividad y realización personal se destaca su inserción laboral que fue mejorando conforme avanzó la intervención. Finalmente en el aspecto organizativo, se incrementó la realización de tareas cotidianas y la funcionalidad en diversas áreas.

La discapacidad relacionada al TOC se disminuyó notablemente lográndose una mayor funcionalidad en las áreas individual, familiar, social, productiva y de autorrealización. Dicha discapacidad es especialmente relevante en la etapa infanto-juvenil y adulta, ya que representa un grave impacto en el funcionamiento social, escolar y familiar (11). Sumado a ello, la comorbilidad en el TOC es más frecuente con otros trastornos de ansiedad y síntomas depresivos, como el afecto negativo, la culpabilidad y el autoconcepto negativo (9). La disminución de la discapacidad a un grado leve favorece la recuperación del funcionamiento organizativo y el bienestar familiar (8). 
La deshabituación y control de los pensamientos obsesivos conseguidos a través de la TCC fue fundamental para descatastrofizar y restar importancia a los mismos. Frente a ello, se conoce que la valoración de sesgos cognitivos explicaría mejor la interpretación errónea que hace una persona sobre su pensamiento obsesivo, favoreciendo el control del mismo (12). Por otro lado, este enfoque sirvió para afrontar los síntomas depresivos y ansiosos que se manifestaban de manera intensa en diversas situaciones. Al respecto, se considera que la psicoterapia debe ir acompañada de una medicación como los antidepresivos para contribuir a la disminución de estos síntomas (13). Sin embargo, su suministro exclusivo no es suficiente para combatirlos. Por lo tanto, el tratamiento farmacológico sirve como coadyuvante a la psicoterapia. La misma aumenta su eficacia al emplear métodos como el contacto telefónico que permite afianzar el acompañamiento al paciente con TOC (14).

Por consiguiente, la TCC trata de dotar al paciente con estrategias de control, afronte y descatastrofización de pensamientos obsesivos, negativos y ansiosos. En este sentido, se trata de ayudar a la persona a descubrir las creencias y valoraciones disfuncionales que están en la base de sus obsesiones. Ante la idea obsesiva, el objetivo de la intervención es necesariamente demostrar las escasas probabilidades de que eso ocurra. De esta manera no se pretende "eliminar" los pensamientos, sino disminuir su influencia en el sujeto y así su frecuencia de aparición (15). Las técnicas estuvieron dirigidas a elementos cognitivos y conductuales que mantenían el círculo obsesivo-compulsivo. El que está conformado por la evitación cognitiva de los pensamientos, las compulsiones, la falta de estrategias adecuadas de afrontamiento y la ausencia de actividades gratificantes (15).

En cuanto a la reducción de la sintomatología depresiva asociada al TOC se registraron efectos positivos en la calidad de vida y los estilos de afrontamiento. Al parecer estos efectos se deben al aumento de la adherencia psicoterapéutica y al incremento del bienestar psicológico que le aportaría mayor seguridad al paciente al momento de relacionarse (16). En este sentido se confirma que uno de los trastornos con mayor comorbilidad en el TOC, es el trastorno depresivo. Este se caracteriza por pensamientos negativos del presente, pasado y futuro, abulia, anhedonia, inseguridad en las relaciones sociales y respecto a las propias capacidades en diversos ámbitos (17). Esto se evidencia en la mayoría de pacientes con TOC que desarrollan episodios de depresión mayor. De modo similar, casi la mitad de pacientes con depresión reportan síntomas obsesivocompulsivos (17). La asociación entre ambos trastornos se caracteriza por la presencia de un curso crónico, donde se pueden presentar internamientos e intentos suicidas $(5,17)$.

En el ámbito familiar y social se mejoró la relación interpersonal, la comunicación y el desempeño de habilidades sociales. Este aspecto coincide con lo reportado por la literatura (16) ya que una mejora en los síntomas patológicos conlleva a mejores resultados en la calidad de vida y el bienestar familiar (12). La efectividad de la TCC en las relaciones familiares puede deberse a la frecuencia semanal de las sesiones ya que asegura el mantenimiento de resultados (18). De esta forma, investigaciones señalan que la terapia familiar cognitivo-conductual para el TOC proporciona un alivio a largo plazo a nivel individual y grupal $(18,19)$. Igualmente, enfocarse en la disfunción familiar puede mejorar el pronóstico a largo plazo (19).

En cuanto a la productividad y realización personal se destaca su inserción laboral. Los déficits hallados en esta área estuvieron relacionados a la alteración de los procesos cognitivos como el pensamiento, atención, memoria, concentración, etc. (20). Este déficit cognitivo limitó la capacidad organizativa, productiva y laboral de la persona. Además, los problemas hallados en la función ejecutiva se ajustan a lo encontrado por estudios neuropsicológicos que señalan una disminución en las habilidades de los pacientes con TOC para realizar tareas que impliquen organización y memoria (21). En este sentido, la aplicación de la TCC ha evidenciado un aumento del rendimiento en la función ejecutiva y en la memoria verbal a corto y largo plazo (22). En efecto, mejora la velocidad del procesamiento de la información, la capacidad de fijar y cambiar el foco de atención, la fluidez verbal y la memoria a corto y largo plazo (21).

Cabe destacar que los resultados favorables de la intervención psicológica se deben a la adherencia psicoterapéutica y al entrenamiento en técnicas cognitivo-conductuales de acuerdo a las situaciones y necesidades de la paciente. Por otro lado, a frente a las recaídas dependerá del paciente poner en práctica las técnicas aprendidas. Asimismo, la funcionalidad 
podría ser influenciada por el apoyo social percibido, el cual puede verse limitado ante el resurgimiento de obsesiones o síntomas depresivos. Por consiguiente, se recomendó a la paciente la búsqueda inmediata de ayuda psicológica sin esperar a que los síntomas de la recaída se agraven.

Se recomienda para futuros estudios clínicos la consideración de técnicas de otros enfoques psicológicos que también puedan ayudar a reducir los síntomas obsesivos, depresivos y ansiosos. Igualmente, se sugiere realizar estudios de casos y controles que permitan la medición y comparación de los resultados. A nivel práctico, es importante difundir la creación de grupos de autoayuda conformados por personas con TOC ya que estos son un medio para fortalecer las técnicas psicoterapéuticas, la capacidad de afrontamiento y las habilidades sociales. En relación al sistema de atención en salud mental se hace necesario, para preservar la dignidad de las personas con trastornos mentales, desarrollar intervenciones bajo un enfoque integral que las ayuden a afrontar por sí mismas la discapacidad derivada de estos. Este sistema debe asegurar que los profesionales de la salud mental sean capacitados en actividades de promoción y detección temprana de los diferentes desórdenes mentales. Finalmente, se concluye que la TCC es una alternativa eficaz para el control y disminución de los síntomas típicos del TOC promoviendo una mayor funcionalidad global en la persona, aminorando la intensidad de los síntomas y los niveles de discapacidad derivados de este trastorno.

\section{REFERENCIAS BIBLIOGRÁFICAS}

1. Kohn R, Rodríguez J. Prevalencia y carga de los trastornos mentales en la población adulta de América Latina y el Caribe. En: Rodríguez J, Kohn R, AguilarGaxiola S, editores. Epidemiología de los trastornos mentales en América Latina y el Caribe. Washington, DC: Organización Panamericana de la Salud; 2009. p. 19-34.

2. Organización Mundial de la salud. Día Mundial de la Salud Mental 2017 - La salud mental en el lugar de trabajo [internet]. Ginebra, Suiza: OMS; 2017 [citado 14 nov 2017]. Disponible en: http://www.who.int/mental_health/world-mentalhealth-day/2017/es/

3. Colombo F. Día Mundial de la Salud Mental: Expertos analizaron los nuevos desafíos que enfrenta la salud mental en las Américas. En: Salud mental, nuevos desafíos para las Américas. Washington, DC:
Organización Panamericana de la Salud, Organización Mundial de la Salud; 2014.

4. Asociación Americana de Psiquiatría. Suplemento del Manual Diagnóstico y Estadístico de Trastornos Mentales, quinta edición. Virginia: American Psychiatric Association Publishing; 2016.

5. Organización Mundial de la salud. Prevención del suicidio un instrumento para médicos generalistas. Ginebra: Departamento de Salud Mental y Toxicomanías; 2000. p. 10.

6. Instituto Nacional de Salud Mental. Estudio epidemiológico de salud mental en Lima Metropolitana y Callao replicación 2012. Informe general. Lima: Anales de Salud Mental; 2013.

7. Vega-Galdós F. Situación, avances y perspectivas en la atención a personas con discapacidad por trastornos mentales en el Perú. Anales de Salud Mental. 2011;27(2):29-32.

8. Huamán J, Alburquerque F, Guillén R. Prevalencia de la discapacidad psiquiátrica en la población adulta de Lima metropolitana y Callao 2002. Anales de Salud Mental. 2015;31(1):45-58.

9. Beck A. Depression cause and treatment. 1a ed. Philadelphia: University of Pennsylvania Press; 1970.

10. Vargas L, Palacios L, González G, De la Peña F. Trastorno obsesivo-compulsivo en niños y adolescentes: una actualización. Segunda parte. Salud mental. 2008;31(4):283-289.

11. Nezu A, Nezu C, Lombardo E. Formulación de casos y diseño de tratamientos cognitivo-conductuales. Un enfoque basado en problemas. $2^{\mathrm{a}}$ ed. México: Editorial El Manual Moderno; 2006.

12. Behobi W, García F, Fernández H. Psicoterapia cognitiva individual del TOC. Salud Mental. 2013;36(4):347-354.

13. Saval J. Exposición y prevención de respuesta en el caso de una joven con trastorno obsesivocompulsivo. Revista de Psicología Clínica con Niños y Adolescentes. 2015;2(1):75-81.

14. Rosa A, Iniesta M, Rosa A. Eficacia de los tratamientos cognitivo-conductuales en el trastorno obsesivo-compulsivo en niños y adolescentes: una revisión cualitativa. Anales de Psicología. 2012;28(2):313-326.

15. Márquez R, Romero T, Fernández M, Ortega M. Guía para la intervención psicológica en el trastorno obsesivo-compulsivo [internet]. Madrid: Facultad de Psicología, Universidad Autónoma de Madrid, s.f.

16. Riveros A, Cortázar-Palapa J, Alcázar F, SánchezSosa J. Efectos de una intervención cognitivoconductual en la calidad de vida, ansiedad, depresión y condición médica de pacientes diabéticos e hipertensos 
esenciales. International Journal of Clinical and Health Psychology. 2005;5(3):445-462.

17. Kodysz S. TrastornoObsesivo-Compulsivo (T.O.C.). Breve Revisión Bibliográfica. Hojas Clínicas de Salud Mental. s.f.

18. Storch E, Geffken G, Merlo L, Mann G, Duke D, Munson M, et. al. Family-Based CognitiveBehavioral Therapy for Pediatric ObsessiveCompulsive Disorder: Comparison of Intensive and Weekly Approaches. Journal of the American Academy of Child \& Adolescent Psychiatry. 2007;46(4):469-478.

doi:

doi.org/10.1097/chi.0b013e31803062e7

19. Barrett P, Farrell L, Dadds M, Boulter N. Cognitive-Behavioral Family Treatment of Childhood Obsessive-Compulsive Disorder: Long-Term Followup and Predictors of Outcome. Journal of the American Academy of Child \& Adolescent Psychiatry. 2005;44(10):1005-1014. doi: doi.org/10.1097/01.chi.0000172555.26349.94

20. Zhu C, Yu F, Ye R, Chen X, Dong Y, Li D, et. al. External Error Monitoring in Subclinical. ObsessiveCompulsive Subjects: Electrophysiological Evidence from a Gambling Task. PloS ONE. 2014;9(3):e90874. doi: 10.1371/journal.pone.0090874

21. Martínez-González A, Piqueras J, Molina J, Lara M. Efectos de una intervención psicológica en la memoria, capacidad de aprendizaje y función ejecutiva de un joven-adulto con TOC. RevDisCliNeuro. 2015;2(1):1-10.

22. Martínez-González A, Piqueras-Rodríguez J. Eficacia de la terapia cognitivo-conductual en trastornos afectivos y de ansiedad mediante neuroimagen funcional. RevNeurol. 2010;50(03):167178. 
ISSN 2519-0652 CASUS.2017;2(3):200-208

\section{Disability in obsessive-compulsive disorder. Beyond a clinical diagnosis}

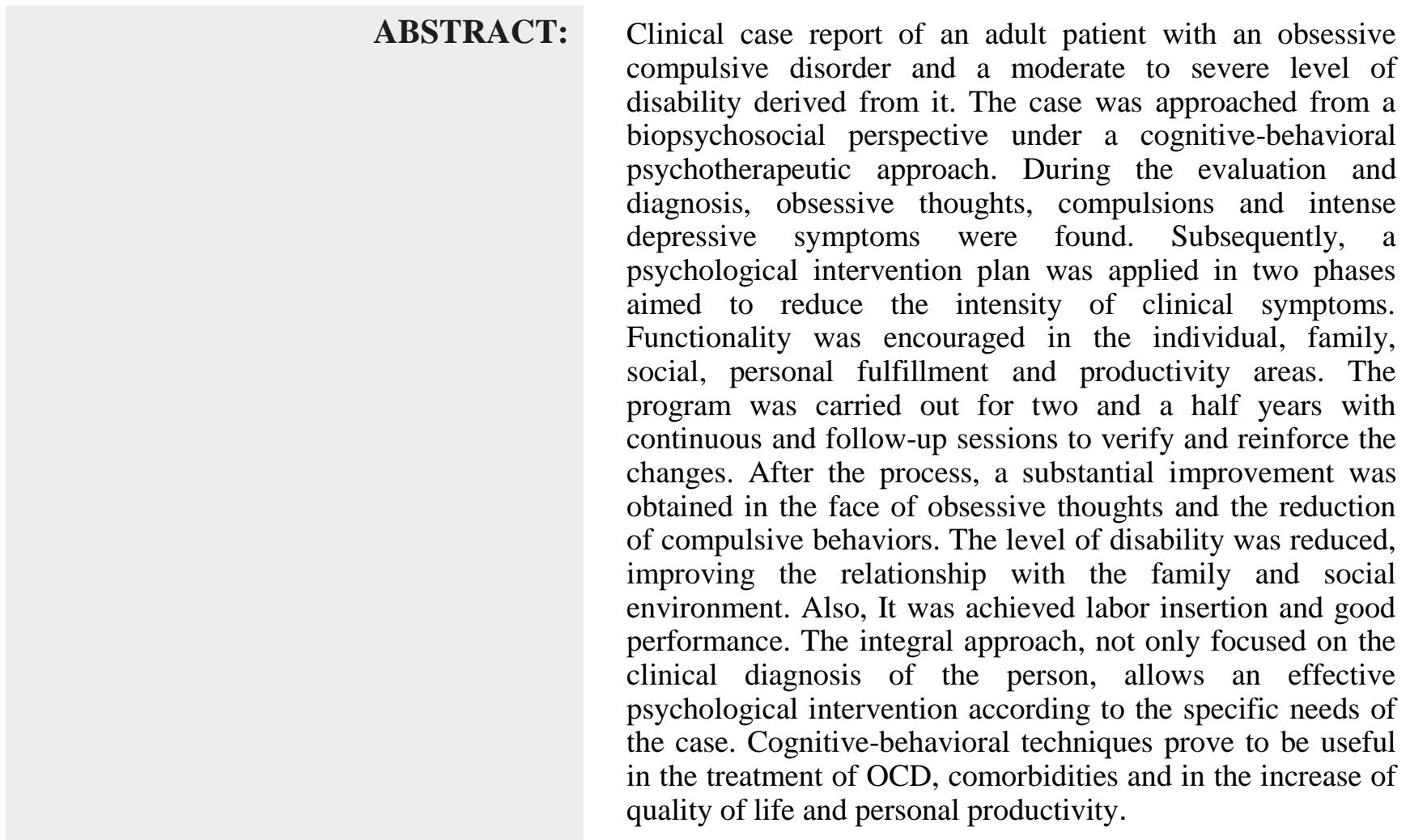

KEY WORDS: Mental disability, Obsessive-compulsive disorder; Cognitive behavioral therapy; Biopsychosocial perspective. 